\title{
Reconfigurable Antennas For Very Wide Spectrum Monitoring
}

\author{
A. Amiri , C. Borda Fortuny, and K. F. Tong \\ Department of Electronic and Electrical Engineering \\ University College London \\ Torrington Place, London, WC1E 7JE, UK \\ k.tong@ucl.ac.uk
}

\begin{abstract}
Two different reconfigurable techniques for spectrum-monitoring antenna are presented. The first method inserts high-performance RF-MEMS switches along the microstrip feed line and back slot of a Vivaldi antenna for switching between three frequency bands centered at $2,3.5$ and 5.25 GHz. The second method collocates three different excitations in the defined space and utilise a common aperture for the bands from $500 \mathrm{MHz}$ to $8 \mathrm{GHz}$. The radiation patterns of the two proposed antennas are maintained in the broadside direction. The proposed antennas offer improved performance over multiple antennas, wideband and multiband application and are suitable for wide $\mathrm{RF}$ spectrum monitoring.
\end{abstract}

\section{Keywords - reconfigurable; multiband, wideband}

\section{I. INTRODUCTION}

Wide RF spectrum monitoring demands antennas with wide operation bandwidth, high and stable radiation pattern across the bands. Frequency reconfigurable antennas are a feasible solution since they can operate in one frequency band while rejecting other bands, thereby increasing signal-to-noise ratio (SNR) [1]. There are basically three design approaches for achieving antenna frequency agility which are as follows:

- antennas integrated with electronic switches, mechanical actuators, tunable materials for reconfigurability in terms of circuital characteristics and/or radiation properties

- reconfigurable/multiband antennas where the same aperture is utilized for different individual radiators.

- ultrawideband (UWB) or multiband antennas integrated with tunable filters

In this paper, we present two high-gain triple-band reconfigurable antennas with stable radiation patterns across the passbands. Although some reconfigurable antennas have been reported, most of them are for narrow band operation [2]. The use of travelling wave antennas, such as Vivaldi antennas, allows working in a wide range of frequencies. Some work has been reported on Vivaldi reconfigurable antennas in [3]-[4]. However the gain of the antennas is low, not suitable for spectrum monitoring.

In the shared aperture antenna approach, interleaved excitations with separate feeds are used. This concept can be utilized for multi-frequency, multi-function, and multipolarization applications. The advantage of shared aperture antenna is possibility to perform multiple tasks simultaneously, achieve narrow beam width, avoid amplitude tapering, and so forth. The main challenges related to shared aperture antenna is to design individual elements which satisfies their requirements and placing the elements on the same aperture while avoiding any physical overlapping and minimize the mutual coupling between excitations.

\section{ANTENNA GEOMETRIES AND DESIGN}

\section{A. Antenna $A$}

Antenna A is designed on a single microwave substrate, as shown in Fig. 1. The dielectric substrate used is Taconic RF-43 with thickness $0.79 \mathrm{~mm}$. The top layer is the microstrip feedline of the antenna. The transition from microstrip to slot line is formed by two quarter-wave stubs. Two RF-MEMS switches are introduced in each stub. The positions of these switches determine the effective length of the stubs, as shown in equation (1), where $\lambda_{\mathrm{o}}$ is the free-space wavelength, $\lambda_{\mathrm{g}}$ is the guided wavelength in the microstrip line, HB indicates the high band and MB indicates the middle band. These distances are optimized for maximum signal coupling at the transition at each reconfigurable band.

$$
\begin{array}{rlrl}
\mathrm{L}_{1} & =\frac{\lambda_{\mathrm{g}, \mathrm{HB}}}{4}, & \mathrm{~L}_{2}=\frac{\lambda_{\mathrm{g}, \mathrm{MB}}}{4}, \\
\mathrm{~L}_{3}=\frac{3}{4} \lambda_{\mathrm{o}, \mathrm{HB}}, & \mathrm{L}_{4}=\frac{3}{4} \lambda_{\mathrm{o}, \mathrm{MB}}
\end{array}
$$

\section{B. Antenna B}

Antenna B consists of interleaving three different excitations in a defined space where the overall aperture is utilised for each individual waveguide. The low band (LB) antenna, i.e. from $500 \mathrm{MHz}$ to $4 \mathrm{GHz}$, is a double-ridged pyramidal horn antenna where it is optimised to improve the performance at $500 \mathrm{MHz}$. The (middle band) MB antenna, i.e. from 4 to $6 \mathrm{GHz}$ is based on a wideband planar monopole to waveguide transition. The high band (HB) antenna, i.e. from 6 to $8 \mathrm{GHz}$, is coaxial cable to waveguide transition conical horn antenna. The arrangement of these three excitations is shown in Fig. 2.

\section{RESULTS AND DISCUSSION}

The simulated $\mathrm{S}_{11}$ parameter and gain at broadside direction, i.e. $(\theta, \phi)=\left(90^{\circ},-90^{\circ}\right)$ of Antenna $A$ at its different operating bands are shown in Fig. 3. The gain is stable within each band and drops rapidly in the immediate proximity of the ends of the band. As the electrical size of the 
radiating aperture varies with operating frequency, the maximum gain variation is $2.6 \mathrm{~dB}$ within a band.

Fig. 4 shows the E- and H-plane radiation patterns of Antenna A. The direction of the main lobe is constant in all bands. The $3 \mathrm{~dB}$ beamwidth is decreasing significantly in $\mathrm{H}$ plane, but it is stable in the E-plane.

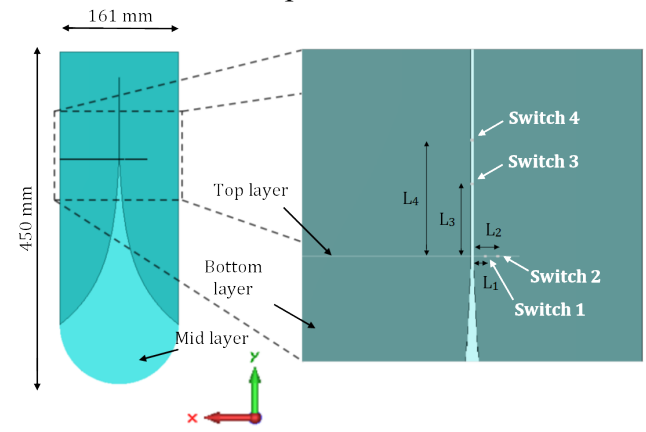

Fig. 1: Geometry of Antenna A and switches position

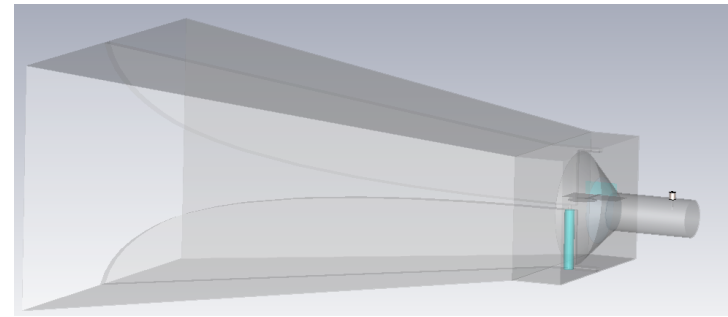

Fig. 2: Geometry of Antenna B

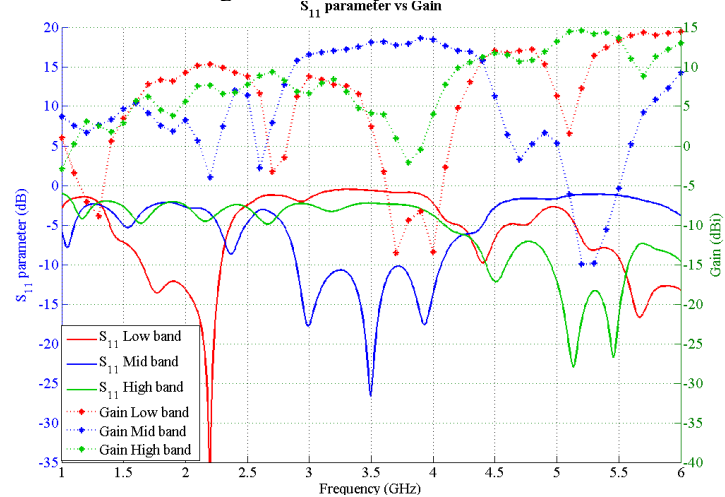

Fig. 3 - Simulated $\mathrm{S}_{11}$ parameter and realized gain of Antenna A

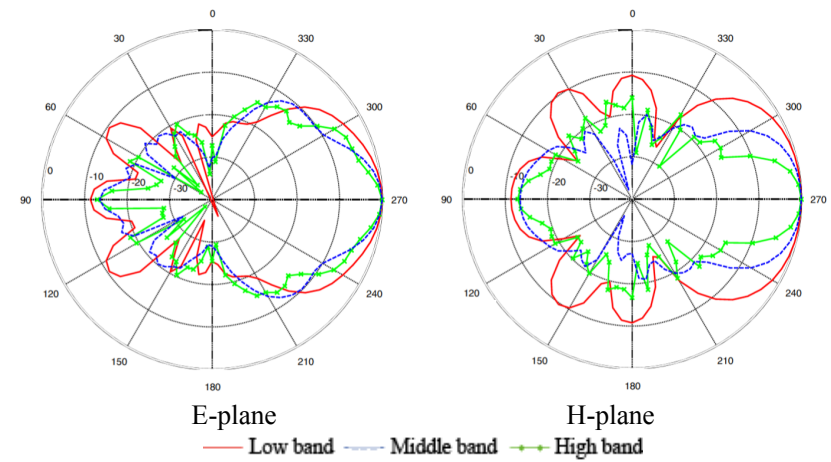

Fig. 4 - Simulated radiation pattern for all bands of Antenna A.

The simulated $\mathrm{S}_{11}$ of Antenna $\mathrm{B}$ is shown in Fig. 5. Three different frequecy bands can be observed. By using different ports of the antenna, different operating bands can be obtained. The isolations between the bands are all below -30 $\mathrm{dB}$. Stable and broadside radiation patterns can be observed in Fig. 6. The gain gradually increases from 5.3 to $17 \mathrm{dBi}$ across $500 \mathrm{MHz}$ and $8 \mathrm{GHz}$. The simulated results of the two antennas are provided by CST Microwave Studio 2013 $[5]$.

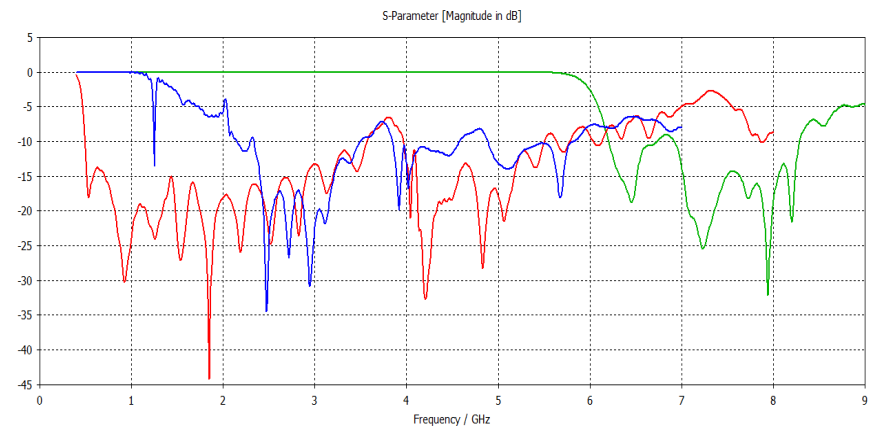

Fig. 5 - Simulated $S_{11}$ parameter and realized gain of Antenna B

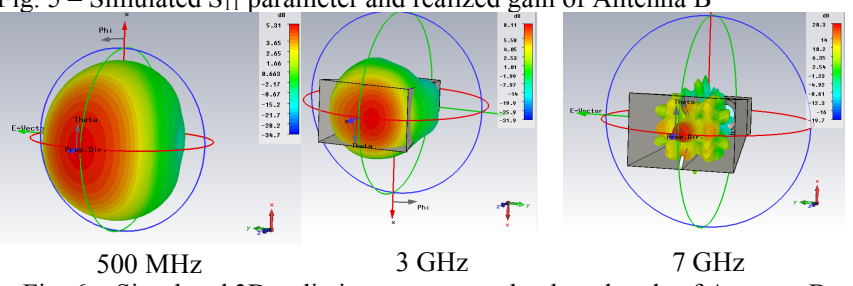

Fig. 6 - Simulated 3D radiation patterns at the three bands of Antenna B

\section{CONCLUSION}

Two triple band reconfigurable antennas based on a Vivaldi antenna and shared aperture concept are presented. RF-MEMS switches are used to tune the Vivaldi antenna to the different operational bands. The switches can be placed in different positions, where other configurable frequency bands can be created for accommodating different system requirements. This design minimizes the effects of the switches because only two switches are working at the same time. The high isolation in the shared aperture geometry allows the antenna to simultaneously operate, i.e. transmit and receive signals, across the wide frequency band. The proposed antennas are currently being fabricated and will be tested to validate the simulation results. The measured results will be presented in the conference.

\section{REFERENCES}

[1] C. Christodoulou, Y. Tawk, S. Lane, and S. Erwin, "Reconfigurable antennas for wireless and space applications," Proceedings of the IEEE, vol. 100, no. 7, pp. 2250-2261, 2012.

[2] J. T. Bernhard, "Reconfigurable antennas and apertures: state of the art and future outlook," pp. 1-9, 2003. [Online]. Available: http://dx.doi.org/10.1117/12.497433

[3] M. Hamid, P. Gardner, P. Hall, and F. Ghanem, "Switched-band vivaldi antenna," Antennas and Propagation, IEEE Transactions on, vol. 59, no. 5, pp. 1472-1480, 2011.

[4] M. R. Hamid, P. Gardner, P. S. Hall, and F. Ghanem, "Reconfigurable vivaldi antenna with tunable stop bands," Microwave and optical technology letters, 52(4), 785-787, 2011.

[5] CST Microwave Studio 2013 User Manual. 